\title{
Spin studies of the short-range correlations at Nuclotron
}

\author{
Vladimir Ladygin* \\ Joint Institute for Nuclear Research, Dubna, Russia \\ E-mail: vladyginejinr.ru
}

V.P. Ladygin ${ }^{1}$, A.V. Averyanov ${ }^{1}$, E.V. Chernykh ${ }^{1}$, D. Enache ${ }^{2}$, Yu.V. Gurchin ${ }^{1}$, A.Yu. Isupov ${ }^{1}$, M. Janek ${ }^{3}$, J.-T. Karachuk ${ }^{1,2}$, A.N. Khrenov ${ }^{1}$, D.O. Krivenkov ${ }^{1}$, P.K. Kurilkin ${ }^{1}$, N.B. Ladygina ${ }^{1}$, A.N. Livanov ${ }^{1}$, O.Mezhenska ${ }^{4}$, S.M. Piyadin ${ }^{1}$, S.G. Reznikov ${ }^{1}$, Ya.T. Skhomenko ${ }^{1,5}$, A.A. Terekhin ${ }^{1}$, A.V. Tishevsky ${ }^{1}$, T. Uesaka ${ }^{6}$ (DSS Collaboration)

${ }^{1}$ Joint Institute for Nuclear Research, Dubna, Russian Federation

${ }^{2}$ National Institute for $R \& D$ in Electrical Engineering ICPE-CA, Bukharest, Romania

${ }^{3}$ Physics Department, University of Žilina, Žilina, Slovak Republic

${ }^{4}$ P.-J̆. Shafarik University, Koshiče, Slovak Republic

${ }^{5}$ Belgorod State National Research University, Belgorod, Russian Federation

${ }^{6}$ Nishina Center for Accelerator-Based Science, RIKEN, Wako, Japan

\begin{abstract}
The results on the angular dependencies of the vector $A_{y}$ and tensor $A_{y y}$ and $A_{x x}$ analyzing powers in deuteron-proton elastic scattering at large scattering angles are presented. These data were obtained at internal target at JINR Nuclotron in the energy range 400-1800 MeV using polarized deuteron beam from new polarized ion source. New data on the deuteron analyzing powers in in the wide energy range demonstrate the sensitivity to the short-range spin structure of the isoscalar nucleon-nucleon correlations. The perspectives of further studies of the short-range correlations using polarized deuteron and proton beams are discussed.
\end{abstract}

23rd International Spin Physics Symposium - SPIN2018 -

10-14 September, 2018

Ferrara, Italy

\footnotetext{
* Speaker.
} 


\section{Introduction}

One of the tools to investigate the Equation-Of-State (EOS) of dense nuclear matter is the study of the Short Range Correlations (SRC) of nucleons in nuclei which is the subject of intensive theoretical and experimental works during last years. Since SRC have densities comparable to the density in the center of a nucleon which is about $\rho \sim 5 \rho_{0}\left(\rho_{0} \approx 0.17 \mathrm{fm}^{-3}\right)$, they can be considered as the drops of cold dense nuclear matter [1]. These studies explore a new part of the phase diagram and very essential to understand the evolution of neutron stars.

The results obtained at BNL [2], SLAC [ [3] and JLAB [4, 可] clearly demonstrate that more than $90 \%$ all nucleons with momenta $k \geq 300 \mathrm{MeV} / c$ belong to two-nucleon (2N) SRC; the probability for a given proton with momenta $300 \leq k \leq 600 \mathrm{MeV} / c$ to belong to $p n$ correlation is $\sim 18$ times larger than for $p p$ correlations; the probability for a nucleon to have momentum $\geq 300 \mathrm{MeV} / c$ in medium nuclei is $\sim 25 \%$; three-nucleon (3N) SRC are present in nuclei with a significant probability [6]. However, still many open questions persist and further investigations are required both from the experimental and theoretical sides. For instance, the experimental data on the spin structure of $2 \mathrm{~N}(\mathrm{I}=1)$ and $3 \mathrm{~N}$ SRC are almost absent.

The main tools to study SRCs at hadronic facilities can be deuteron structure investigations at large internal momenta allowing to explore $2 \mathrm{~N}$ SRC with $I=0 ;{ }^{3} \mathrm{He}$ structure to understand the role of $2 \mathrm{~N}$ SRC with $I=1$ and $3 \mathrm{~N}$ SRC; nuclei breakup $A(p, p p) X, A(p, p n) X, A(p, p p p) X$ etc. with the detection of few nucleons in the final state. The greate importance is the study of the spin effects in these reactions because the data on the SRCs spin structure are scarce. Nuclotron and NICA will allow to investigate the spin effects for multi-nucleon correlations in a wide energy range.

The main goal of the Deuteron Spin Structure (DSS) experimental program is to obtain the information on the spin - dependent parts of $2 \mathrm{~N}$ and $3 \mathrm{~N}$ forces from two processes: $d p$ - elastic scattering in a wide energy range and $d p$-nonmesonic breakup with two protons detection at energies $300-500 \mathrm{MeV}$ [7, 8, 9] using the Nuclotron Internal Target Station (ITS) [10]. The motivation of this program at low and intermediate energies is based on theoretical analysis of the experimental results obtained for the deuteron induced reactions (see recent reviews [11, 12] and references therein). Importance of the $d p$ - elastic scattering studies at high energy is discussed in [13].

Such experimental program at Nuclotron was started by the measurements of the vector $A_{y}$ and tensor $A_{y y}$ and $A_{x x}$ analyzing powers in $d p$ - elastic scattering at $T_{d}$ of $880 \mathrm{MeV}$ [14] and $2000 \mathrm{MeV}$ [15]. The systematic measurements of the differential cross section have been performed also in recent years [16, 17, 18].

In this paper we report new results of the energy scan of the vector $A_{y}$ and tensor $A_{y y}$ and $A_{x x}$ analyzing powers in $d p$ - elastic scattering obtained at the Nuclotron ITS [10] in the energy range of $400-1800 \mathrm{MeV}$.

\section{Experiment at Nuclotron ITS}

The ITS setup is well suited for study of the energy dependence of polarization observables for the deuteron-proton elastic scattering and deuteron breakup reaction with the detection of two protons at large scattering angles. For these purposes the $\mathrm{CH}_{2}$-target of $10 \mu \mathrm{m}$ thick is used for 
the measurements. The yield from carbon content of the $\mathrm{CH}_{2}$-target is estimated in separate measurements using several twisted $8 \mu \mathrm{m}$ carbon wires. The monitoring of the intensity is done from the detection of $p p$ - quasielastic scattering at $90^{\circ}$ in $\mathrm{cms}$ by the scintillation counters placed in the horizontal plane. The detection of the $d p$ - elastic events is done by the coincidence measurements of the proton and deuteron. The detectors are placed in the both horizontal and vertical planes for the analyzing powers measurements. The selection of the $d p$ - elastic events is done by the correlation of the energy losses in plastic scintillators for deuteron and proton and their time-of-flight difference. The use of large amount of the scintillation counters allowed to cover wide angular range [19]. Such a metod has been used to obtain the polarization data in $d p$ - elastic scattering at $T_{d}$ of $880 \mathrm{MeV}$ [14] and $2000 \mathrm{MeV}$ [15].

The upgraded setup at ITS has been used to measure the vector $A_{y}$ and tensor $A_{y y}$ and $A_{x x}$ analyzing powers in $d p$ - elastic scattering between $400 \mathrm{MeV}$ and $1800 \mathrm{MeV}$ using polarized deuteron beam from new Source of Polarized Ions (SPI) developed at LHEP-JINR [20]. These measurements were performed using internal target station at Nuclotron [10] with new control and data acquisition system [21]. The existing setup [19] has been upgraded by new VME based DAQ [22], new MPod based high voltage system [23], new system of the luminosity monitors etc.

The same setup has been used as a polarimeter based on the use of $d p$ - elastic scattering at large angles $\left(\theta_{\mathrm{cm}} \geq 60^{\circ}\right)$ at $270 \mathrm{MeV}[19$, where precise data on analyzing powers [24, 25, 26] exist, has been developed at ITS at Nuclotron [10]. The accuracy of the determination of the deuteron beam polarization achieved with this method is better than $2 \%$ because of the values of the analyzing powers were obtained for the polarized deuteron beam, which absolute polarization had been calibrated via the ${ }^{12} \mathrm{C}(d, \alpha){ }^{10} \mathrm{~B}^{*}\left[2^{+}\right]$reaction [26].

\section{Measurements of the analyzing power in $d p$ - elastic scattering}

New SPI [20] has been used to provide polarized deuteron beam. In the current experiment the spin modes with the maximal ideal values of $\left(P_{z}, P_{z z}\right)=(0,0),(-1 / 3,+1)$ and $(-1 / 3,-1)$ were used. The deuteron beam polarization has been measured at $270 \mathrm{MeV}$ [19]. The $d p$ - elastic scattering events at $270 \mathrm{MeV}$ were selected using correlation of the energy losses and time-of-flight difference for deuteron and proton detectors. The values of the beam polarization for different spin have been obtained as weighted averages for 8 scattering angles for $d p$ - elastic scattering in the horizontal plane only. The typical values of the beam polarization were $\sim 65-75 \%$ from the ideal values [27].

After deuteron beam polarization measurements at $270 \mathrm{MeV}$, the beam has been accelerated up to the required energy $T_{d}$ between $400 \mathrm{MeV}$ and $1800 \mathrm{MeV}$. The scintillation detectors were positioned in the horizontal and vertical plane in accordance with the kinematic of $d p$ - elastic scattering for the investigated energy The main part of the measurements were performed using $\mathrm{CH}_{2}$ target. Carbon target was used to estimate the background. The selection of the $d p$ - elastic events is done by the correlation of the energy losses in plastic scintillators for deuteron and proton and their Time-Of-Flight (TOF) difference those initial distributions are shown in the left bottom and upper panels in Fig. 1, respectively. The right upper panel in Fig. 1 demonstrates the TOF difference for proton and deuterons with prompt TOF window, while the final selection of the $d p$ elastic events after applying a graphical cut on the correlation of the energy losses is shown in the right bottom panel. Additionally, the criteria on the beam-target interaction point has been applied 
(Fig. 2). The normalized numbers of $d p$-elastic scattering events for each spin mode were used to calculate the values of the analyzing powers $A_{y}, A_{y y}$ and $A_{x x}$.
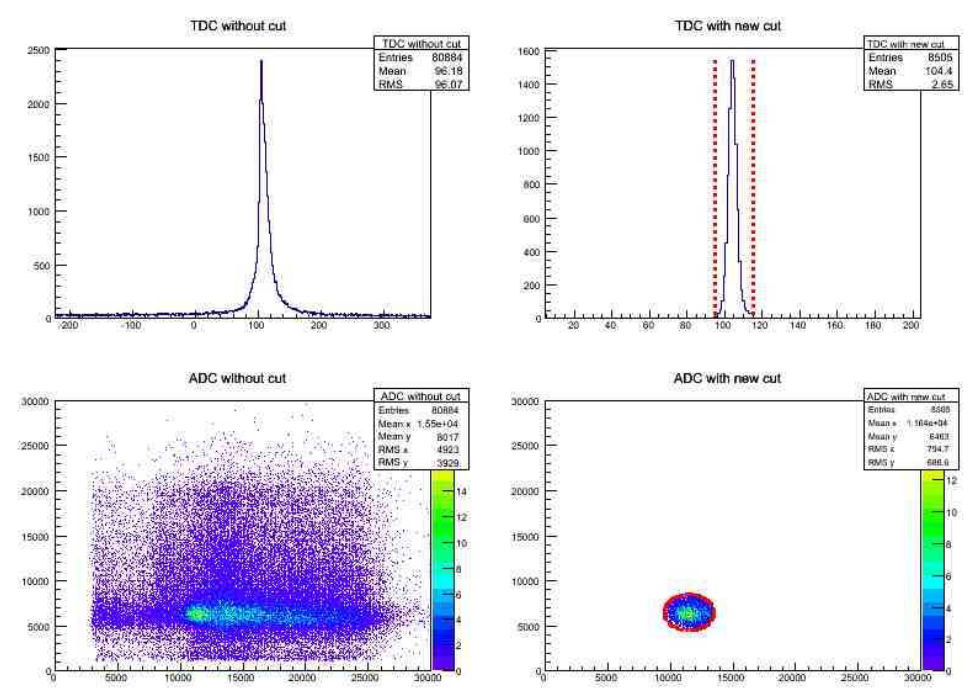

Figure 1: Selection of the $d p$-elastic events at $\sim 75^{\circ}$ in $\mathrm{cms}$ at $1000 \mathrm{MeV}$ using time difference and correlation of the energy-losses signals signals for proton and deuteron counters.

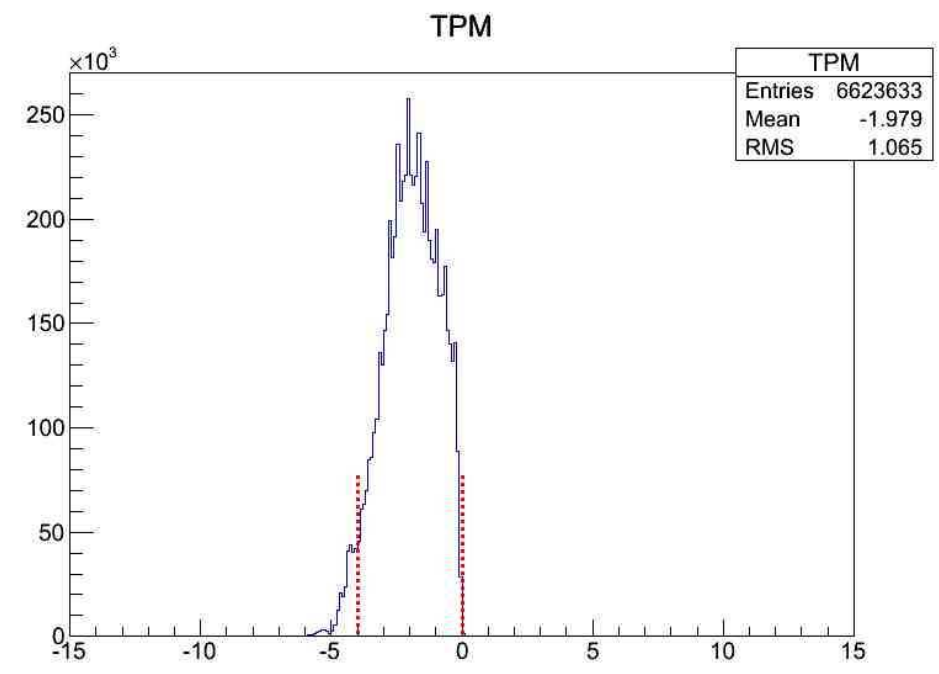

Figure 2: Distribution of the beam-target interaction point. The vertical lines represent the criteria to select the $d p$-elastic scattering events.

The angular dependencies of the vector $A_{y}$, tensor $A_{y y}$ and $A_{x x}$ analyzing powers at the deuteron kinetic energy $T_{d}$ of $400 \mathrm{MeV}$ are presented in Fig. 3, Fig. 6 and Fig. 5, respectively. The full squares are the results of the DSS experiment at ITS at Nuclotron. Open squares, triangles and circles are the data obtained at IUCF [28], [29] and at Saclay [30], respectively. One can see good agreement of new data obtained at Nuclotron with the data from earlier experiments [28]-[30]. The theoretical calculations were performed in the relativistic multiple scattering expansion formalism 


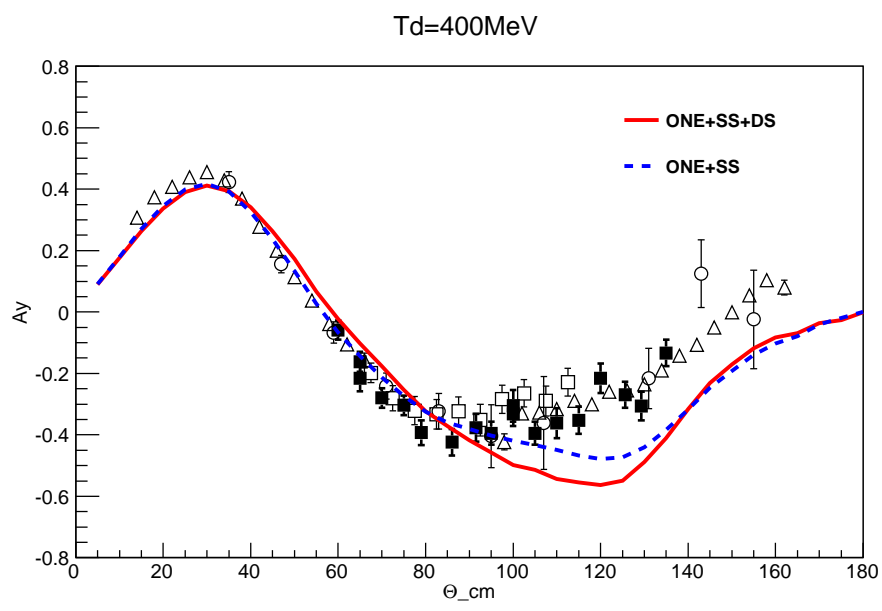

Figure 3: The angular dependence of the vector analyzing power $A_{y}$ at the deuteron kinetic energy $T_{d}$ of $400 \mathrm{MeV}$. The full squares are the preliminary results of the DSS experiment at ITS at Nuclotron. Open symbols are the world data [28, 29, 30]. Curves are explained in the text.

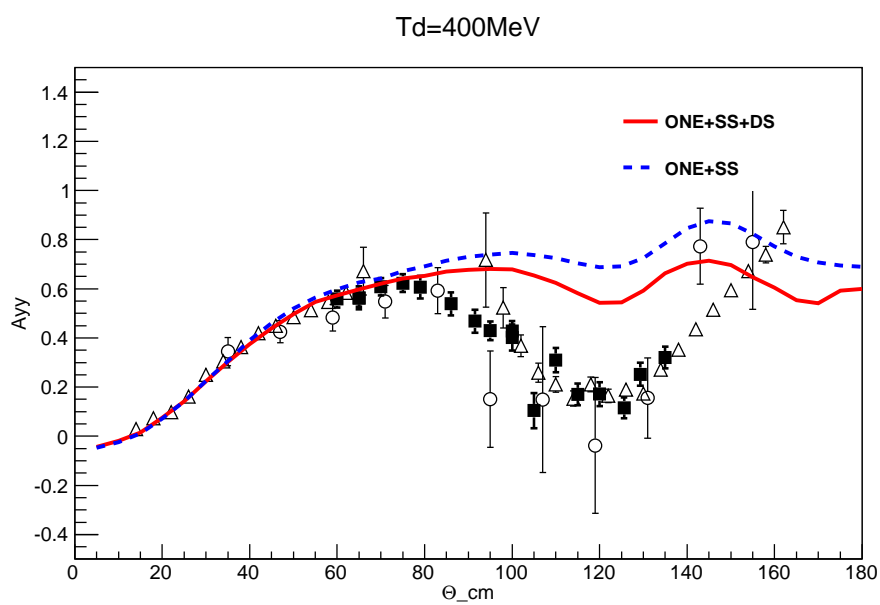

Figure 4: The angular dependence of the tensor analyzing power $A_{y y}$ at the deuteron kinetic energy $T_{d}$ of $400 \mathrm{MeV}$. Symbols and lines are the same as in Fig. B.

[31]-[33]. The four contributions are taken into account: One-Nucleon-Exchange (ONE), Singleand Double- Scattering (SS and DS), and $\Delta$ - isobar excitation. The presented approach was applied earlier to describe the differential cross sections at deuteron energies between 500 and $1300 \mathrm{MeV}$ in a whole angular range [33]. Dashed and solid lines are the calculations performed within relativistic multiple scattering model [31]-[32] considering ONE+SS terms only and with the DS contribution added, respectively. Note that the contribution of the $\Delta$ - isobar mechanism is negligible at this energy [33]. The relativistic multiple scattering model [31]-[32] describes the data on $A_{y}$ and $A_{y y}$ up to $\sim 90^{\circ}$ only, while it fails to reproduce the data at larger angles. The considering of the DS term does not improve the agreement. The $A_{x x}$ behaviour is not described by the model [31]-[32] over the whole angular range. The considering of the contribution of the three-nucleon forces or $\mathrm{N}^{4} \mathrm{LO}$ calculations performed within chiral Effective Field Theory ( $\chi$ EFT) [34] do not allow to get 


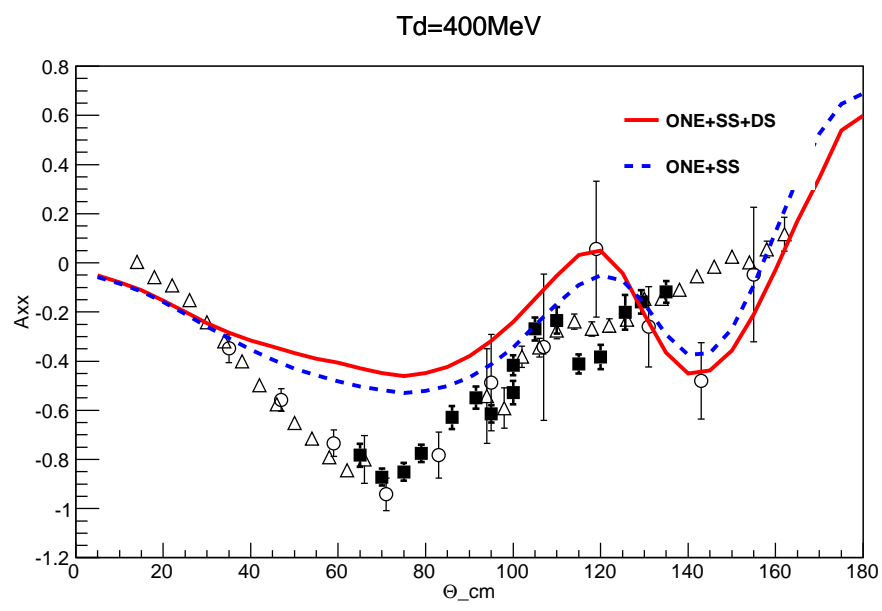

Figure 5: The angular dependence of the tensor analyzing power $A_{x x}$ at the deuteron kinetic energy $T_{d}$ of $400 \mathrm{MeV}$. Symbols and lines are the same as in Fig. 3 .

an agreement with the data on the tensor analyzing powers. The reason of the deviation can be the neglecting by the $3 \mathrm{~N}$ SRCs.

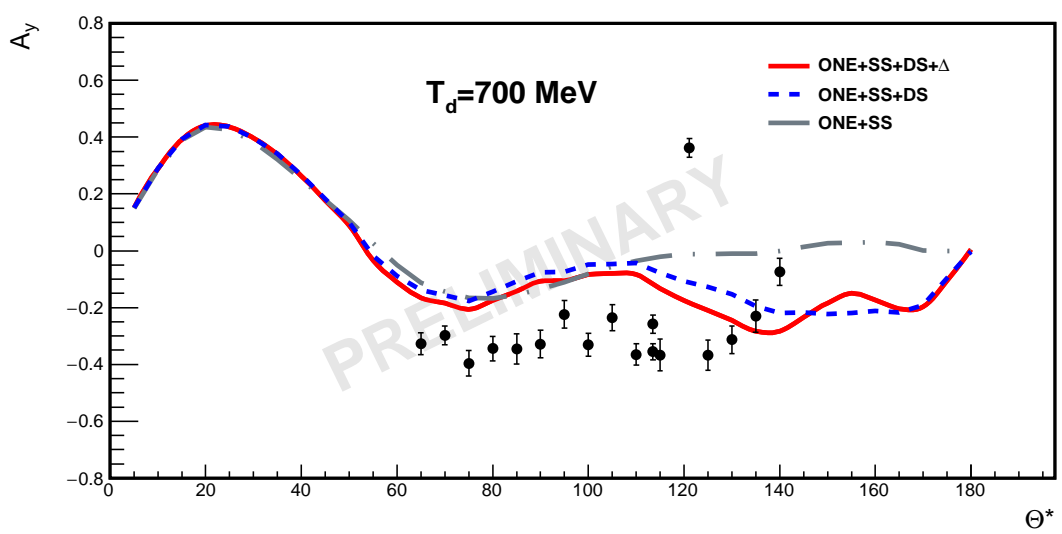

Figure 6: The angular dependence of the vector analyzing power $A_{y}$ at the deuteron kinetic energy $T_{d}$ of $800 \mathrm{MeV}$. The full symbols are the preliminary results of the DSS experiment at ITS at Nuclotron. Curves are explained in the text.

The preliminary results on the vector $A_{y}$, tensor $A_{y y}$ and $A_{x x}$ analyzing powers at the deuteron kinetic energy $T_{d}$ of $800 \mathrm{MeV}$ are presented in Fig. 6, Fig. 7 and Fig. 8, respectively. The data are obtained within angular range of $65^{\circ}-140^{\circ}$ in cms. The dash-dotted, dashed and solid lines are the predictions obtained within relativistic multiple scattering model [33] considering ONE+SS terms only, with the DS contribution and with $\Delta$ - isobar excitation term, respectively. One can see, that the DS- term consideration allows to improve the agreement with the data on $A_{y y}$ analyzing power at the angles up to $\sim 120^{\circ}$. The contribution of $\Delta$ - isobar excitation is small even at backward angles. Analyzing power $A_{y}$ and $A_{x x}$ are not described by the calculations. The similar picture in the description of the data on the analyzing power is observed at $700 \mathrm{MeV}$. 


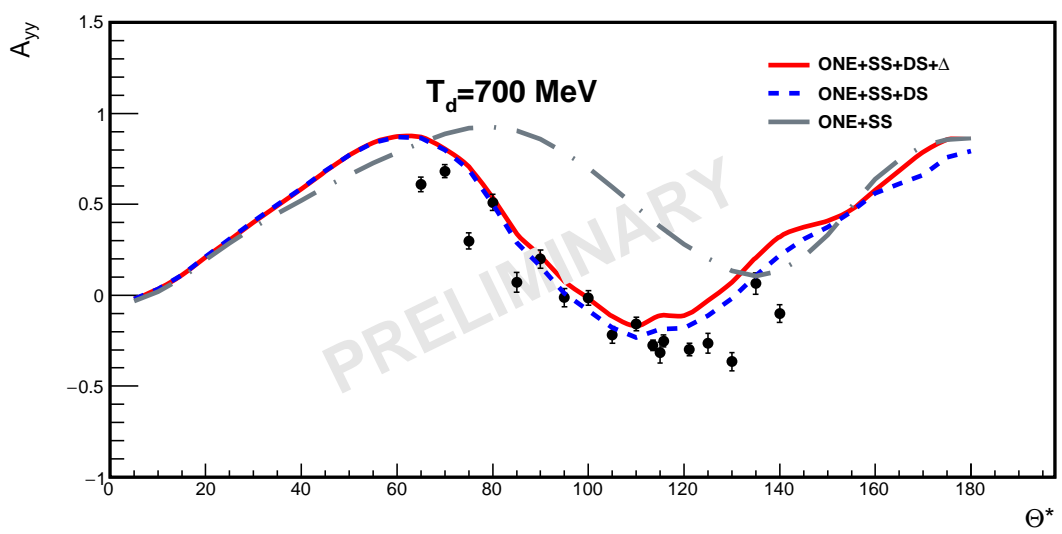

Figure 7: The angular dependence of the tensor analyzing power $A_{y y}$ at the deuteron kinetic energy $T_{d}$ of $800 \mathrm{MeV}$. Lines and symbols are the same as in Fig. 6 .

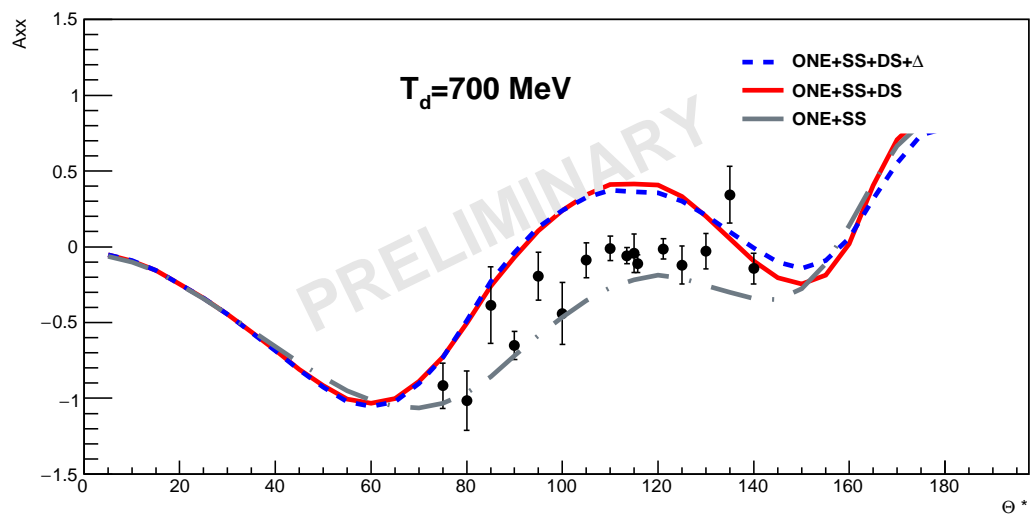

Figure 8: The angular dependence of the tensor analyzing power $A_{x x}$ at the deuteron kinetic energy $T_{d}$ of $800 \mathrm{MeV}$. Lines and symbols are the same as in Fig. 6.

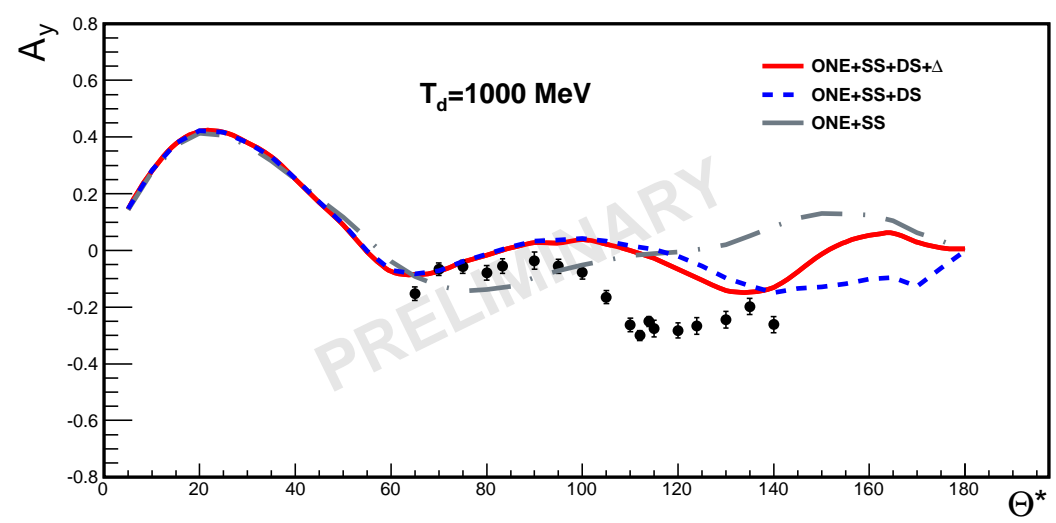

Figure 9: The angular dependence of the vector analyzing power $A_{y}$ at the deuteron kinetic energy $T_{d}$ of $1000 \mathrm{MeV}$. The full symbols are the preliminary results of the DSS experiment at ITS at Nuclotron. Lines are the same as in Fig. 6 . 


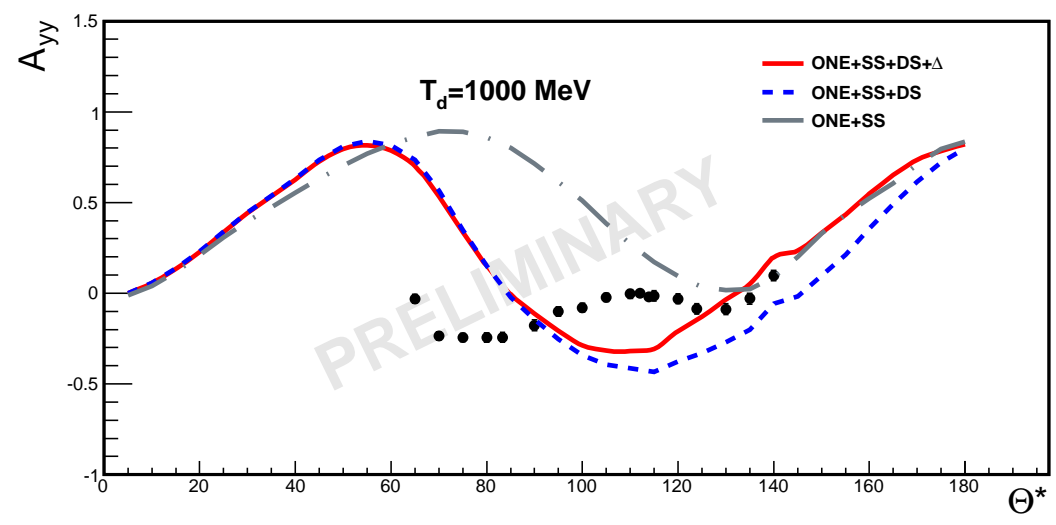

Figure 10: The angular dependence of the tensor analyzing power $A_{y y}$ at the deuteron kinetic energy $T_{d}$ of $1000 \mathrm{MeV}$. Lines and symbols are the same as in Fig. 6 .

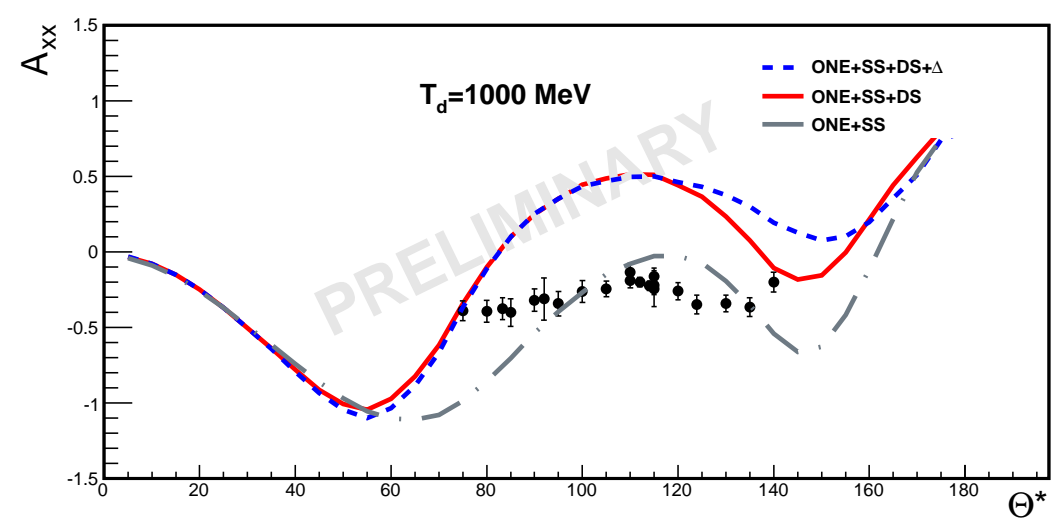

Figure 11: The angular dependence of the tensor analyzing power $A_{x x}$ at the deuteron kinetic energy $T_{d}$ of $1000 \mathrm{MeV}$. Lines and symbols are the same as in Fig. 6 .

The preliminary results on the vector $A_{y}$, tensor $A_{y y}$ and $A_{x x}$ analyzing powers at the deuteron kinetic energy $T_{d}$ of $1000 \mathrm{MeV}$ are presented in Fig. 9, Fig. 10 and Fig. 11, respectively. The lines are the predictions obtained within relativistic multiple scattering model [33] considering ONE, SS, DS and $\Delta$ - isobar excitation terms. They are the same as in Fig. 6. One can see that the model describes the behavior of the vector analyzing power $A_{y}$ up to $\sim 100^{\circ}$ in $\mathrm{cms}$, while the tensor analyzing powers $A_{y y}$ and $A_{x x}$ are not described over whole range of measurements. The $\Delta$ - isobar excitation term gives a significant contribution at the angles larger than $140^{\circ}$ in cms. Apparently, spin structure of the nucleon-nucleon interactions and deuteron at short distances is missed in the standard description used in the relativistic multiple scattering model [31]-[33].

The energy dependencies of the vector $A_{y}$ and tensor $A_{y y}$ analyzing powers at $70^{\circ}$ in the cms are presented as a function of the transverse momentum $P_{T}$ in Figs 12 and 13 , respectively. The full circles are the preliminary results of the present experiment. The full squares are the data obtained at ITS at Nuclotron in 2005 [14]-[15]. Open symbols are the world data [24, 25, 30, 35, 36]. Both $A_{y}$ and $A_{y y}$ analyzing powers change the sign at $P_{T} \sim 600 \mathrm{MeV} / c$ and have the tendencies at 


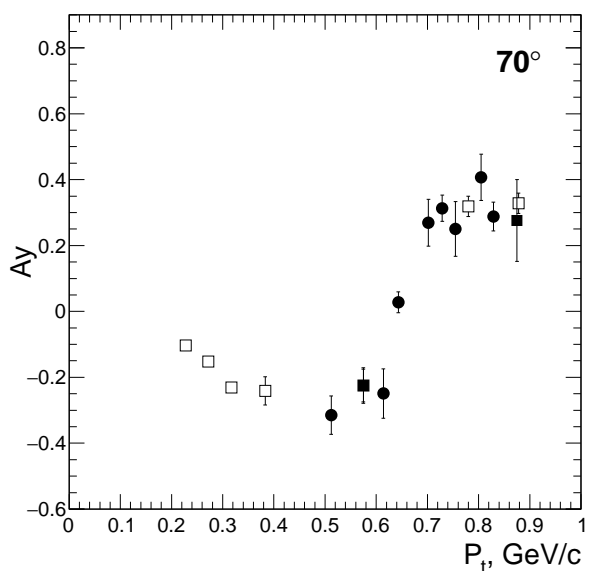

Figure 12: The energy dependence of the vector analyzing power $A_{y}$ at $70^{\circ}$ in the cms. The full circles are the preliminary results of the present experiment. The full squares are the data obtained at ITS at Nuclotron in 2005 [14]-[15]. Open symbols are the data $[24,25,30,35,36$ obtained in the previous experiments.

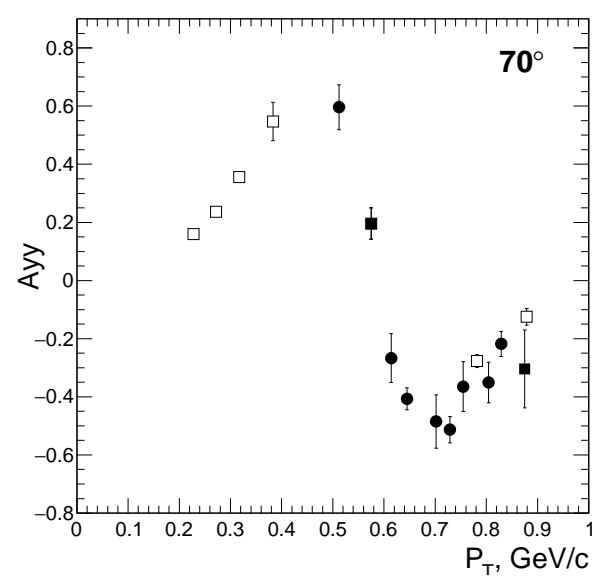

Figure 13: The energy dependence of the tensor analyzing power $A_{y y}$ at $70^{\circ}$ in the cms. The full circles are the preliminary results of the present experiment. The full squares are the data obtained at ITS at Nuclotron in 2005 [14]-[15]. Open symbols are the same as in Fig. 12 .

larger $P_{T}$ to reach the positive and negative constant values, respectively. These features of the data indicate the serious deviation of the spin structure of the 2N SRCs on the standard description of the nucleon-nucleon interaction. Further theoretical investigations are required to understand the behaviour of the data at large $P_{T}$.

The availability of the polarized proton beam at Nuclotron [37] allows to extend the DSS physics program at ITS [13], namely, to perform the experiments on the measurements of the nucleon analyzing power $A_{y}^{p}$ in $p d$ - elastic scattering at $135-1000 \mathrm{MeV}$ and in $p d$-non-mesonic breakup at the energies between $135-250 \mathrm{MeV}$ for different kinematic configurations etc.

\section{Conclusions}

- Upgraded Nuclotron with new SPI [20] provides quite unique opportunity for the studies of the spin effects and polarization phenomena in few body systems.

- The realization of the DSS program at ITS will allow to obtain the crucial data on the spin structure of 2-nucleon and 3- nucleon short range correlations. The first natural step in these studies, namely, the energy scan of the deuteron analyzing powers in $d p$-elastic scattering has been performed in 2016-2017. The data demonstrate the sensitivity to the short-range spin structure of the deuteron.

- Next experiments using polarized deuterons and protons at ITS are in preparation.

- The extension of the studies to the high energies is possible with the extracted polarized deuteron and proton beams. 
The authors thank the Nuclotron staff for providing good conditions of the experiment. They thank A.S. Belov, V.B. Shutov and V.V Fimushkin for the tune of the SPI 20]. They express the gratitude to S.N. Bazylev, V.I. Maximenkova, I.V. Slepnev, V.M. Slepnev and A.V. Shutov for the help during the preparation of the detector and DAQ system. The work has been supported in part by the RFBR under grants $N^{0} 16-02-00203 \mathrm{a}$ and $N^{0} 19-02-00079$ a, by the Ministry of Education, Science, Research, and Sport of the Slovak Republic (VEGA Grant No. 1/0113/18), by JINRSlovak Republic and JINR-Romania scientific cooperation programs in 2016-2018.

\section{References}

[1] L. Frankfurt, M. Sargsian, M. Strikman, Recent observation of short range nucleon correlations in nuclei and their implications for the structure of nuclei and neutron stars, Int. J. Mod. Phys. A23 (2008) 2991.

[2] E. Piasetzky, M. Sargsian, L. Frankfurt, M. Strikman, J.W. Watson, Evidence for the strong dominance of proton-neutron correlations in nuclei, Phys. Rev. Lett. 97 (2006) 162504.

[3] L. L. Frankfurt, M. I. Strikman, D. B. Day, M. M. Sargsian, Evidence for short range correlations from high $Q^{2}\left(e, e^{\prime}\right)$ reactions, Phys. Rev. C48 (1993) 2451.

[4] K. Sh. Egiyan et al., Observation of nuclear scaling in the $A\left(e, e^{\prime}\right)$ reaction at $x(B)$ greater than 1 , Phys. Rev. C68 (2003) 014313.

[5] K. S. Egiyan et al., Measurement of 2- and 3-nucleon short range correlation probabilities in nuclei, Phys. Rev. Lett. 96 (2006) 082501.

[6] L. Frankfurt, M. Sargsian, and M. Strikman, Future directions for probing two and three nucleon short-range correlations at high energies AIP Conf. Proc. 1056 (2008) 322.

[7] V. P. Ladygin et al., Spin physics in few body systems at Nuclotron, Phys. Part. Nucl. 45 (2014) 327.

[8] V. P. Ladygin et al., Few-body Studies at Nuclotron-JINR, Few Body Syst. 55 (2014) 709.

[9] M. Janek et al., Investigation of the dp breakup and dp elastic reactions at intermediate energies at Nuclotron, Few Body Syst. 58 (2017) 40.

[10] A. I. Malakhov et al., Potentialities of the internal target station at the Nuclotron, Nucl. Instrum. Meth. in Phys. Res. A440 (2000) 320.

[11] W. Glöckle, H. Witala, D. Hüber, H. Kamada, J. Golak, The Three nucleon continuum: achievements, challenges and applications, Phys. Rep. 274 (1996) 107.

[12] N. Kalantar-Nayestanaki, E. Epelbaum, J. G. Messchendorp and A. Nogga, Signatures of three-nucleon interactions in few-nucleon systems, Rep. Prog. Phys. 75 (2012) 016301.

[13] V. P. Ladygin et al., The spin studies in few-body systems at Nuclotron Int. J. Mod. Phys. Conf. Ser. 40 (2016) 1660074.

[14] P. K. Kurilkin et al., Measurement of the vector and tensor analyzing powers for dp-elastic scattering at $880 \mathrm{MeV}$, Phys. Lett. B715 (2012) 61.

[15] P. K. Kurilkin et al., Investigation of the angular dependence of the analyzing powers in the deuteron-proton elastic scattering at the nuclotron, Phys. Part. Nucl. Lett. 8 (2011) 1081.

[16] Yu. V. Gurchin et al., The cross-section in dp-elastic scattering at the energies of $500 \mathrm{MeV}, 700 \mathrm{MeV}$ and $880 \mathrm{MeV}$ obtained at the internal target station of Nuclotron, Phys. Part. Nucl. Lett. 10 (2013) 243. 
[17] A. A. Terekhin et al., Study of the dp-elastic scattering at 2 GeV, Phys. Part. Nucl. Lett. 12 (2015) 695.

[18] A. A. Terekhin et al., Differential cross section for elastic deuteron - proton scattering at the energy of $700 \mathrm{MeV}$ per nucleon, Phys. Atom. Nucl. 80 (2017) 106.

[19] P. K. Kurilkin et al., The $270 \mathrm{MeV}$ deuteron beam polarimeter at the Nuclotron Internal Target Station, Nucl. Instr. Meth. in Phys. Res. $\mathbf{A 6 4 2}$ (2011) 45.

[20] V. V. Fimushkin et al., Development of polarized ion source for the JINR accelerator complex, J. Phys. Conf. Ser. 678 (2016) 012058;

A. S. Belov et al., Source of polarized ions for the JINR accelerator complex, J. Phys. Conf. Ser. 938 (2017) 012017.

[21] A. Yu. Isupov, V. A. Krasnov, V. P. Ladygin, S. M. Piyadin, S. G. Reznikov, The Nuclotron internal target control and data acquisition system, Nucl. Instrum. Meth. in Phys. Res. $\mathbf{A 6 9 8}$ (2013) 127.

[22] A. Yu. Isupov, Online polarimetry of the Nuclotron internal deuteron and proton beams, J. Phys. Conf. Ser. 938 (2017) 012019.

[23] Ya. T. Skhomenko et al., Software development for multi-channel LV/HV supply system based on Wiener MPod, BSU Sci. Bull. Math.\& Phys. 234 (2016) 126.

[24] K. Sekiguchi et al., Complete set of precise deuteron analyzing powers at intermediate energies: comparison with modern nuclear force predictions, Phys. Rev. C65 (2002) 034003.

[25] K. Sekiguchi et al., Polarization transfer measurement for ${ }^{1} H(\vec{d}, \vec{p})^{2} H$ elastic scattering at 135-MeV/u and three nucleon force effects, Phys. Rev. C70 (2004) 014001.

[26] K. Suda et al., Absolute calibration of the deuteron beam polarization at intermediate energies via the ${ }^{12} \mathrm{C}(d, \alpha){ }^{10} \mathrm{~B}^{*}\left[2^{+}\right]$reaction, Nucl. Instr. Meth. in Phys. Res. $\mathbf{A 5 7 2}$ (2007) 745.

[27] Ya. T. Skhomenko et al., Measurement of the deuteron beam polarization at internal target at Nuclotron for DSS experiment, J. Phys. Conf. Ser. 938 (2017) 012022.

[28] R. V. Cadman et al., Evidence for a three nucleon force effect in proton - deuteron elastic scattering, Phys. Rev. Lett. 86 (2001) 967.

[29] B. v Przewoski et al., Analyzing powers and spin correlation coefficients for $p+d$ elastic scattering at $135 \mathrm{MeV}$ and $200 \mathrm{MeV}$, Phys. Rev. $\mathbf{C 7 4}$ (2006) 064003.

[30] M. Garçon et al., Measurements of vector and tensor analysing powers for 191 and $395 \mathrm{MeV}$ deuteron scattering, Nucl. Phys. A458 (1986) 287.

[31] N. B. Ladygina, Deuteron-proton elastic scattering at intermediate energies, Phys. Atom. Nucl. 71 (2008) 2039.

[32] N. B. Ladygina, Differential cross section of dp-elastic scattering at intermediate energies, Eur. Phys. J. A42 (2009) 91.

[33] N. B. Ladygina, Delta excitation in deuteron- proton elastic scattering, Eur. Phys. J. A52 (2016) 199.

[34] S. Binder et al., Few-nucleon systems with state-of-the-art chiral nucleon-nucleon forces, Phys. Rev. C93 (2016) 044002.

[35] M. Haji-Saied et al., Tensor and vector spin observables in pd elastic scattering at $600 \mathrm{MeV}, 800$ MeV, and $1000 \mathrm{MeV}$, Phys. Rev. C36 (1987) 2010.

[36] V. Ghazikhanian et al., Vector and tensor spin observables in the reaction ${ }^{1} \vec{H}(\vec{d}, d)^{1} \vec{H}$ at $1.6 \mathrm{GeV}$, Phys. Rev. C43 (1991) 1532. 
[37] V. P. Ladygin et al., First results on the measurements of the proton beam polarization at internal target at Nuclotron, J. Phys. Conf. Ser. 938 (2017) 012008. 